\title{
Chemical Biology in the Embryo: In Situ Imaging of Sulfur Biochemistry in Normal and Proteoglycan-Deficient Cartilage Matrix
}

\author{
Mark J. Hackett, ${ }^{\dagger}$ Graham N. George, ${ }^{*, \dagger, \ddagger, \S}$ Ingrid J. Pickering, ${ }^{\dagger,}, \S$ and B. Frank Eames ${ }^{*, \|}$ \\ ${ }^{\dagger}$ Molecular and Environmental Sciences Group, Department of Geological Sciences, University of Saskatchewan, Saskatoon, \\ Saskatchewan S7N 5E2, Canada \\ ${ }^{\ddagger}$ Department of Chemistry, University of Saskatchewan, Saskatoon, Saskatchewan S7N 5C9, Canada \\ ${ }^{\S}$ Toxicology Centre, University of Saskatchewan, Saskatoon, Saskatchewan S7N 5B3, Canada \\ "Department of Anatomy and Cell Biology, University of Saskatchewan, Saskatoon, Saskatchewan S7N 5E5, Canada
}

\section{Supporting Information}

\begin{abstract}
Proteoglycans (PGs) are heavily glycosylated proteins that play major structural and biological roles in many tissues. Proteoglycans are abundant in cartilage extracellular matrix; their loss is a main feature of the joint disease osteoarthritis. Proteoglycan function is regulated by sulfation-sulfate ester formation with specific sugar residues. Visualization of sulfation within cartilage matrix would yield vital insights into its biological roles. We present synchrotron-based X-ray fluorescence imaging of developing zebrafish cartilage, providing the first in situ maps of sulfate ester distribution. Levels of both sulfur and sulfate esters decrease as cartilage develops through late phase differentiation (maturation or hypertrophy), suggesting a functional link between cartilage matrix sulfur content and chondrocyte differentiation. Genetic experiments confirm that sulfate ester levels were due to cartilage proteoglycans and support the hypothesis that sulfate ester levels regulate chondrocyte differentiation. Surprisingly, in the PG synthesis mutant, the total level of sulfur was not significantly reduced, suggesting sulfur is distributed in an alternative chemical form during lowered cartilage proteoglycan production. Fourier transform infrared imaging indicated increased levels of protein in the mutant fish, suggesting that this alternative sulfur form might be ascribed to an increased level of protein synthesis in the mutant fish, as part of a compensatory mechanism.
\end{abstract}

$\mathrm{P}$ roteoglycans (PGs) are heavily glycosylated proteins that play a critical role in both development and disease. During development of the vertebrate skeleton, for example, abundant PG secretion in the extracellular matrix provides function to both cartilage and bone. ${ }^{1}$ Defects in PG synthesis and homeostasis can lead to skeletal disease. A main feature of the joint disease osteoarthritis is loss of PGs in cartilage matrix. ${ }^{2}$ PG function is regulated by sulfation, during which sulfates are added to specific sugar residues as esters. Sulfation of PGs is important for at least two biological roles of cartilage matrix that are relevant to defects in osteoarthritis patients: tissue integrity and growth factor signaling. ${ }^{2,3}$ The amount of PG sulfation in cartilage matrix is thought to be directly proportional to how much water it absorbs, which determines the compression-resistant strength of cartilage. ${ }^{4}$ In addition to this structural role, PG sulfation influences binding affinities (and thus signaling activity) of growth factors and is required for normal growth factor signaling in cartilage matrix. ${ }^{5,6}$ In fact, many of the studies of the signaling role of cartilage PGs originate from genetic perturbations to PG sulfation pathways. $^{7-12}$ Despite the importance of sulfated PGs, methods for their visualization are quite limited. Histological stains, such as Alcian blue or Safranin O, can reflect PG sulfation; however, the mechanisms through which the dye binds are unclear and can be nonspecific. ${ }^{13}$ Moreover, although antibodies have been generated against sulfated PGs, these may be too specific as an antibody might sense only one isomer of a particular sulfated PG. ${ }^{14}$ We present herein methods for direct visualization of sulfur chemical forms, and specifically sulfate esters, in situ within developing cartilage matrix through the use of chemically specific X-ray fluorescence imaging (XFI). ${ }^{15}$

XFI is a powerful method that can be used to map the distribution of elements at the regional, cellular, and subcellular levels in biological samples. ${ }^{15}$ The technique has found numerous applications across the field of analytical biochemistry to study the anatomical distribution of elements ${ }^{15}$ in tissue samples as diverse as bone ${ }^{16,17}$ and brain. ${ }^{18}$ While other methods such as proton-induced X-ray emission spectroscopy and scanning electron microscopy have been used previously to investigate the distribution of total sulfur in cartilage, ${ }^{19-23}$ these techniques lack XFI's combination of sensitivity to low concentrations and high spatial resolution. In addition, XFI, when properly used, can also provide chemically specific imaging, which we have exploited for the first time in this study to image sulfate esters. Chemically specific XFI takes advantage of the difference in X-ray energies required to excite different chemical forms of sulfur. The chemical shift range at the sulfur

Received: October 17, 2015

Revised: $\quad$ March 10, 2016

Published: March 17, 2016 
K-edge spans a relatively wide energy range (approximately 14 $\mathrm{eV}$ ), reflecting the diversity of sulfur chemical forms and oxidation states present in biological tissues (Figure $S 1$ and Table S1). Hence, several XFI measurements of the same sample can be taken, each at a slightly different incident energy corresponding to excitation energies of different sulfur chemical forms. This contrasts with XFI elemental mapping, for which the energy of the incident X-ray beam is typically set well above the excitation energy of a wide range of elements, allowing multiple elements to be imaged simultaneously but without chemical discrimination. Chemically specific imaging of sulfur was first demonstrated by our group in plant tissues ${ }^{24}$ and more recently was attempted for samples of rat brain ${ }^{25}$ and bovine cornea, ${ }^{26}$ although these reports do not correctly discriminate between sulfate esters and inorganic sulfate. We present herein the first chemically specific XFI of sulfate esters in biological samples, with additional high-resolution trace-level XFI of sulfur and phosphorus.

Larval stage zebrafish are used increasingly as an experimental model to understand human development and disease. $^{27,28}$ There are a number of advantages to the zebrafish model, including high fecundity, ease of culture, very well characterized developmental stages, and remarkable conservation of gene function with higher vertebrates. Additionally, a wide variety of mutant and transgenic zebrafish are available, each to elucidate a given gene's function. We have used larval stage zebrafish to test the hypothesis that sulfation levels of cartilage PGs regulate chondrocyte differentiation. As part of the process of endochondral ossification, chondrocytes embed themselves in a PG-rich extracellular matrix and then progress along a differentiation pathway termed maturation or hypertrophy. ${ }^{1,29}$ Histological and biochemical studies have suggested that levels of sulfated PGs in cartilage matrix decrease as chondrocytes mature (Figure 1A). ${ }^{30,31}$ The maturation process has been established to begin from the middle of the cartilage element, and thus, a gradient of mature to immature chondrocytes exists from the center of the cartilage element extending in both directions to the anterior and posterior ends. $^{31-33}$ This predicts that lower levels of both total sulfur and sulfate esters would be present in these regions of developing cartilage. Moreover, we also predict decreased sulfur levels in developing cartilage of PG synthesis mutant zebrafish (Figure 1B,C). ${ }^{32,33}$ Similar to the histologically observed inverse relation between $P G$ sulfation and chondrocyte maturation, ${ }^{30,31}$ PG defective mutant cartilage shows an increased level of maturation. ${ }^{32}$ Our results demonstrate the importance of chemically specific XFI and provide unique insights into the in vivo relationship between PG sulfation and chondrocyte differentiation.

\section{METHODS}

Zebrafish. Zebrafish adults and embryos were maintained in accordance with Canadian animal ethics protocols at the University of Saskatchewan. At 6 days postfertilization, zebrafish larvae were anaesthetized with MESAB (MS-222; $0.02 \%)$, embedded in optimal cutting temperature (OCT) medium, and immediately flash-frozen in liquid nitrogen-cooled iso-pentane. For fam 20 b- PG synthesis, mutant zebrafish larvae $\left(\right.$ fam $\left.20 b^{b 1127}\right)$ heads were embedded in OCT while tails were placed into a DNA lysis solution, and mutants were identified according to the polymerase chain reaction protocol previously described. $^{32}$ Tissue sections, cut at $10 \mu \mathrm{m}$, were melted onto thermanox plastic for XFI analysis, except for subcellular

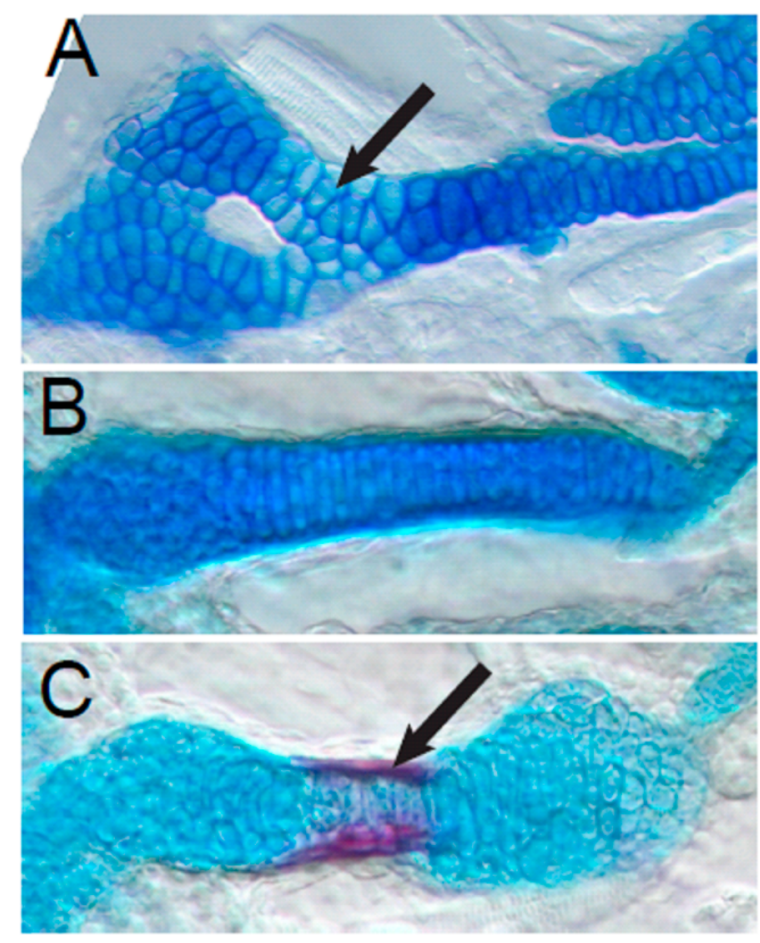

Figure 1. Alcian blue staining suggests a correlation between sulfated proteoglycan levels and chondrocyte differentiation. Regions of cartilage that have mature chondrocytes, such as the middle of this developing zebrafish hyosymplectic (A, arrow), show decreased levels of Alcian blue staining. Compared to wild-type levels of Alcian blue staining of the developing zebrafish ceratohyal (B), proteoglycan synthesis mutants, such as this fam $20 \mathrm{~b}$ mutant, have decreased levels of Alcian blue staining and also early chondrocyte maturation and perichondral bone (C, arrow).

imaging for which micrometer sections were melted onto $\mathrm{Si}_{3} \mathrm{~N}_{4}$ substrate. All tissue sectioning was performed on the same day, on an instrument calibrated within an error of $10 \%$. We have previously used confocal microscopy to measure the thickness of tissue sections cut on this instrument to confirm that the error is within $10 \% .^{34}$ Tissue sections were cut from additional fish and melted onto infrared transparent $\mathrm{CaF}_{2}$ windows for FTIR spectroscopic imaging analysis. All samples were air-dried prior to analyses.

Choice of Flash-Freezing without Formalin Fixation. Previous studies ${ }^{35,36}$ have shown that chemical fixation (i.e., formalin fixation) can drastically alter the elemental distribution and chemical composition of biological samples. In the study presented here, sulfur K-edge XAS was measured on zebrafish sections prepared using different protocols (Figure S3). Formalin fixation followed by plastic embedding (methacrylate embedding) resulted in a significant alteration in sulfur speciation, notably increasing the levels of sulfoxides, and is not recommended as a preparation method for the study sulfur speciation. Cryo-fixation, with or without prior formalin fixation, yielded no significant differences in sulfur speciation. In addition, XFI was used to compare elemental distributions in flash-frozen cartilage with and without formalin fixation (Figure S4). While sulfur levels were unchanged between the two methods, formalin-fixed flash-frozen tissue contained significantly more chlorine than non-formalin-fixed flash-frozen tissue (Figure S4) because of a high $\mathrm{Cl}^{-}$level from the fixation buffer. Hence, flash-freezing tissue samples without formalin fixation was chosen as the sample preparation method. 
Histology. Following XFI analysis, sections were fixed in a $4 \%$ buffered formalin solution ( $\mathrm{pH} 7)$ and stained following a routine hematoxylin and eosin ( $\mathrm{H} \& \mathrm{E})$ protocol, as previously described. ${ }^{34}$ Whole-mount Alcian blue staining of cartilage was conducted as previously described. ${ }^{32}$

XAS Data Collection at Stanford Synchrotron Radiation Laboratory (SSRL) Beamline 4-3. Bulk sulfur K-edge XAS spectra of zebrafish tissue sections and standard solutions were collected using a $\mathrm{Si}(111)$ double-crystal monochromator with an upstream collimating mirror set at an angle sufficient to reject the $\mathrm{Si}(333)$ and higher harmonics. The incident beam was reduced to $1 \mathrm{~mm} \times 3 \mathrm{~mm}$ by vertical and horizontal slits, and the intensity was measured with a helium gas-filled ionization chamber, $I_{0}$. Samples (tissue sections and solutions) were mounted at $45^{\circ}$ to the incident beam, and X-ray fluorescence was collected with a nitrogen gas-filled SternHeald Lytle detector (The EXAFS Co., Pioche, NV). Prior to the collection of spectra, the sample chamber was purged with $\mathrm{He}$ until the relative $\mathrm{O}_{2}$ content within the chamber was $<0.5 \%$. XAS spectra were calibrated against the spectrum of an anhydrous $\mathrm{Na}_{2} \mathrm{~S}_{2} \mathrm{O}_{3}$ powder solid standard, with the lowestenergy peak thought to be $2469.2 \mathrm{eV}$, as described previously. ${ }^{24,37-39}$ Spectra were collected across the energy range of $2450-2515 \mathrm{eV}$, with a total collection time of approximately $5 \mathrm{~min}$, using the XAS-Collect software. ${ }^{40}$ An average spectrum was calculated for each sample from three replicate measurements. Spectra of calibration standards and model compounds were recorded at room temperature. Standard compounds used for fitting routines (Figure S1 and Table S1) represented disulfides (oxidized glutathione), thiols (reduced glutathione), thio-ethers (methionine), sulfoxides (methionine sulfoxide), sulfinic acids (hypotaurine), sulfonic acids (taurine), O-linked sulfate esters ( $\mathrm{N}$-acetyl D-galactosamine 6-sulfate), $\mathrm{N}$-linked sulfate esters (D-galactosamine 2sulfate), and inorganic sulfates $\left(\mathrm{Na}_{2} \mathrm{SO}_{4}\right)$. Standards were analyzed as solutions to minimize the self-absorption artifacts previously reported, ${ }^{38,41}$ made up to $30-100 \mathrm{mM}$ in PBS at $\mathrm{pH}$ 7.4 and measured in sulfur-free custom-made polycarbonate cuvettes with thin polypropylene windows.

XAS Data Collection at the Canadian Light Source (CLS) Soft X-ray Microcharacterization Beamline (SXRMB). Data collection used a configuration similar to that at SSRL beamline 4-3, except that the incident beam was reduced to $2 \mathrm{~mm} \times 4 \mathrm{~mm}$ by vertical and horizontal slits, and the X-ray fluorescence was measured using a four-element $\mathrm{Si}$ drift detector array (Hitachi HHS, USA, Northridge, CA). Spectra were collected at room temperature across the energy range of $2450-2515 \mathrm{eV}$, with a total collection time of approximately $10 \mathrm{~min}$.

XAS Data Analysis and Processing. Spectra were processed using the EXAFSPAK suite of programs. ${ }^{42}$ Using the DATFIT program, spectra collected from tissue sections were fitted with a linear combination of reference spectra (discussed above). Standards were excluded from the refinement algorithm if they contributed to $<0.5 \%$ of the total spectra, at a value $<3$ times their standard deviation of measurement (calculated from the diagonal elements of the variance-covariance matrix).

Elemental Mapping Using XFI. Unless otherwise noted, XFI used a focused beam with the sample mounted at $45^{\circ}$ in a purged helium atmosphere and raster scanned through the incident beam. The incident beam intensity was measured with a helium gas-filled $I_{0}$ ion chamber. Fluorescence emission spectra were recorded with a four-element vortex detector at $90^{\circ}$ to the incident beam.

Regional Spatial Resolution XFI at the CLS VESPERS Beamline. The incident pink beam was focused to a spot size of approximately $4 \mu \mathrm{m} \times 5 \mu \mathrm{m}$ (horizontal $\times$ vertical) using Kirkpatrick-Baez $(\mathrm{KB})$ mirror optics. The sample was raster scanned in $5 \mu \mathrm{m}$ steps with a dwell time of $2 \mathrm{~s}$. The full emission spectrum via multichannel analysis (MCA) was recorded at each pixel, in addition to single channels for $\mathrm{P}, \mathrm{S}$, $\mathrm{Cl}, \mathrm{K}, \mathrm{Ca}, \mathrm{Fe}, \mathrm{Cu}$, and $\mathrm{Zn}$ fluorescence lines, and elastic scatter.

Cellular and Subcellular Spatial Resolution XFI at Advanced Photon Source (APS) Beamline 2-ID-B. The incident beam of $2530 \mathrm{eV}$ from a multilayer spherical grating monochromator was focused to a spot size of $0.1 \mu \mathrm{m}$ using Fresnel zone plate optics. Samples, mounted at $15^{\circ}$ to the incident beam, were raster scanned using a step size (effective pixel size) of either 1 or $0.1 \mu \mathrm{m}$ (cellular or subcellular spatial resolution, respectively), with a dwell time of $100 \mathrm{~ms}$. Fluorescence emission spectra and single channels for P, S, and elastic scatter were collected.

Quantification of XFI Data. To quantify elemental (or chemically specific) sulfur maps, regions of interest (ROIs) were drawn for specific anatomical locations in the cartilage, aided by the overlay of histological images with XFI maps. ROIs were drawn to calculate the average elemental or sulfate ester concentration for the entire ceratohyal $(\mathrm{CH})$ cartilage region and also for posterior and medial regions of the cartilage. The boundaries for the medial region of the cartilage were defined by the high $\mathrm{P}$ content associated with mineralized tissue. ROIs were defined using Sam's Micro-Analysis ToolKit software $^{43}$ for image data collected at CLS VESPERS or at SSRL beamline $14-3$, or using MAPS $^{44}$ software for data collected at APS beamline 2-ID-B.

Sulfur Chemical Species Mapping Using Chemically Specific XFI. Chemically specific XFI requires high energy resolution with good stability and reproducibility as well as a focused beam. This was achieved at regional spatial resolution on SSRL beamline 14-3. KB optics focused the incident beam to a spot size of approximately $5 \mu \mathrm{m} \times 5 \mu \mathrm{m}$ (horizontal $\times$ vertical). The sample was raster scanned through the beam in 3 $\mu \mathrm{m}$ steps, with a dwell time of $1 \mathrm{~s}$. The vortex detector readout was synchronized to the stage movement speed, and data were collected continuously, such that the full emission spectrum was collected every $1 \mathrm{~s}$, for an average stage movement of $3 \mu \mathrm{m}$. Images were collected at incident energies of 2510.0, 2479.8, 2478.2, and $2475.0 \mathrm{eV}$. Energy was calibrated to the lowestenergy peak of anhydrous sodium thiosulfate, thought to be $2469.2 \mathrm{eV}$. Following image collection, micro-XAS spectra were recorded from specific locations using a step size of $0.15 \mathrm{eV}$ with a dwell time of $2 \mathrm{~s}$.

Chemically Specific XFI General Strategy. The process of chemically specific imaging has been previously described in detail. $^{15,24}$ In general, and slightly modified for windowed fluorescence data at the sulfur K-edge, to visualize $n$ different chemical forms of sulfur, $n+2$ fluorescence maps of sulfur are collected. A map is collected with incident energy tuned to the energy of the strongest peak of each of the $n$ chemical forms of sulfur, plus two additional maps below and above the sulfur Kedge, equivalent to a background, non-sulfur fluorescence map and a total sulfur map, respectively. The background fluorescence map is subtracted from each of the remaining $n$ +1 fluorescence maps. Normalized fluorescence intensities are determined from model compound spectra of the $n$ sulfur 

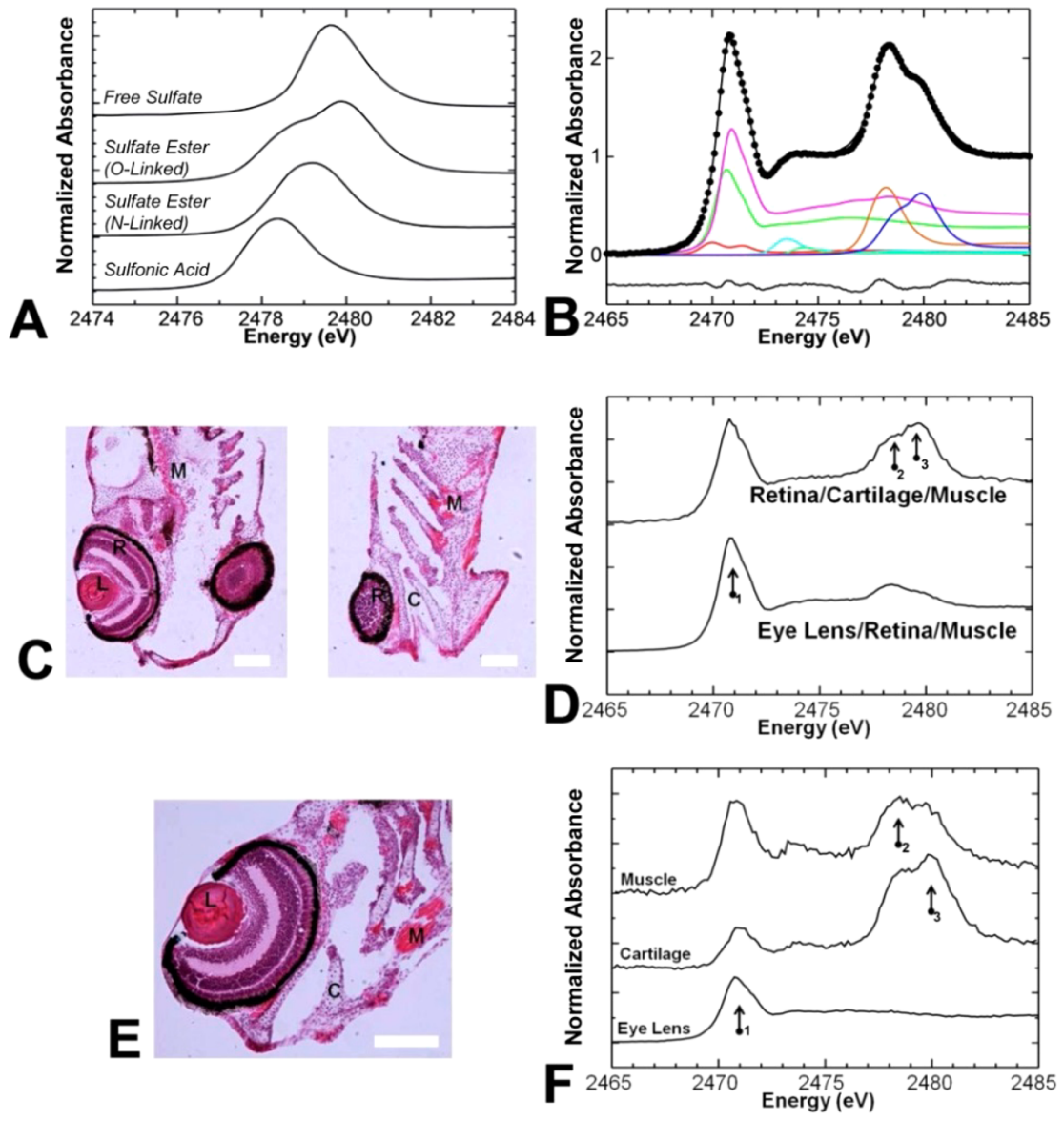

Figure 2. K-Edge of sulfur that is sensitive to oxidation state and chemical form. (A) Sulfur K-edge XAS spectra of model compounds representing sulfur species possible in cartilage. In particular, sulfates have different spectral features depending on their chemical form (free, $\mathrm{N}$-linked ester, or Olinked-ester). (B) Least-squares fits of bulk zebrafish tissues do not detect free or N-linked sulfate, only O-linked sulfate. Color scheme: thiol, green; thio-ether, pink; disulfide, red; sulfoxide, light blue; sulfonic acid, orange; O-linked sulfate ester, dark blue. Raw data are shown as black circles, the fit is shown as a black line. The residual is shown below the spectra. (C and D) Bulk measurements performed on zebrafish tissue sections cut at different planes detect chemical differences that correlate to tissue structures present in the sample (i.e., more sulfate esters when more cartilage is present, more thio-ether when eye lens is present). (E and F) $\mu$-XAS can be used to prove chemical speciation on a fine spatial scale (e.g., $10 \mu \mathrm{m} \times$ $10 \mu \mathrm{m}$ region) and detect chemical differences among eye lens, cartilage, and muscle; locations from which $\mu$-XAS (F) were measured are indicated on the histological image (E). Abbreviations for panels C and E: C, cartilage; L, eye lens; M, muscle; R, retina. Arrows numbered 1-3 in panels E and $\mathrm{F}$ highlight the positions of maximal absorbance due to thio-ethers, sulfonic acids, and sulfate esters, respectively, which are most abundant in the eye lens, muscle tissue, and cartilage tissue, respectively. The scale bar in panel $\mathrm{C}$ is $20 \mu \mathrm{m}$, and the scale bar in panel $\mathrm{E}$ is $50 \mu \mathrm{m}$.

chemical forms. Each pixel intensity is expressed as a linear combination of the normalized intensity of components multiplied by their abundance at that pixel. The simultaneous equations are solved by matrix inversion to calculate maps for the $n$ different chemical forms of sulfur. Dividing by the total sulfur map expresses the contribution of each chemical form of sulfur as the percentage of total sulfur. This approach can image the total sulfur metabolome in a biological sample in a time much shorter than that needed to collect individual spectra per pixel (essentially impractical within a realistic time for dilute species) coupled with least-squares fitting of spectra. However, this process still requires numerous maps (typically six to nine) to image all biologically likely sulfur chemical forms, often resulting in data collection for many hours per sample.

Higher-Excitation Energy Modification of Chemically Specific XFI. Because the current study focused on the distribution of sulfate esters that have a high excitation energy, specific images of chemical components with lower excitation energies such as thiols, thio-ethers, disulfides, and sulfoxides were not needed. Consequently, the protocol was modified to image sulfate esters through collection of four rather than eight maps in half the experimental data collection time. Specifically, the new background map was collected at $2475.0 \mathrm{eV}$ to account for both non-sulfur fluorescence and the fluorescence of lowerexcitation energy sulfur (thiols, disulfides, thio-ethers, and sulfoxides). This decreased the sulfur chemical forms used in imaging $(n=2)$, with maps collected at the strongest peaks for sulfonic acids $(2478.2 \mathrm{eV})$ and sulfate esters $(2479.8 \mathrm{eV})$, and the total sulfur map collected above the sulfur edge (2510.0 $\mathrm{eV})$. The $2475.0 \mathrm{eV}$ map was subtracted from the 2478.2 and $2479.8 \mathrm{eV}$ maps and then matrix inversion applied as previously described. $^{24}$

Validation of a Modified Chemically Specific XFI Protocol. The modified approach assumes that the fluorescence intensity from lower-oxidation state species has negligible variation in the energy range above $2475 \mathrm{eV}$. To test this, a series of spectra were simulated for mixtures of thiols, thio-ethers, disulfides, and sulfoxides across a range of compositions expected in cartilage (Figure S5). While below $2475 \mathrm{eV}$ these spectra show large intensity variations, above 

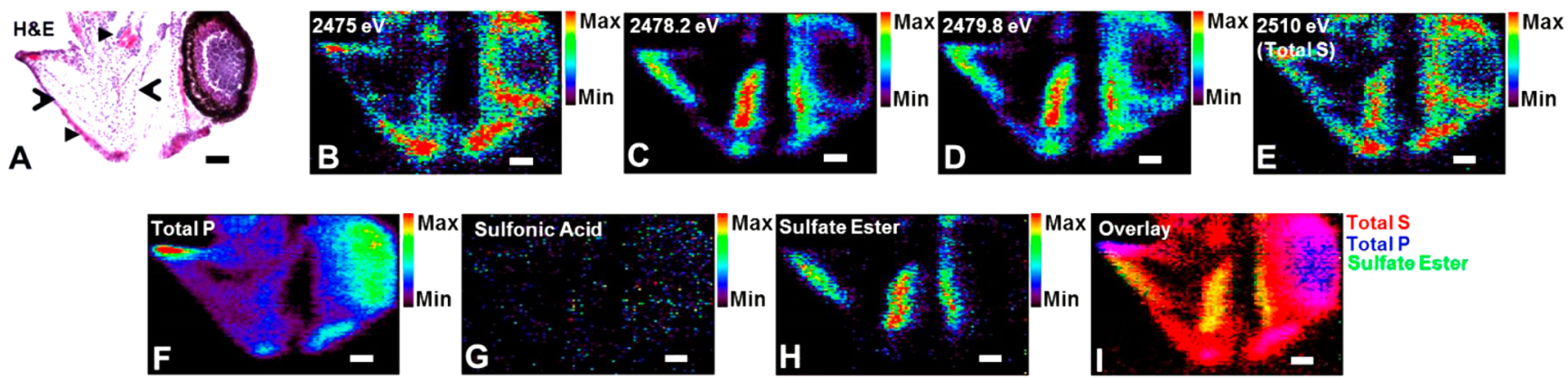

Figure 3. Chemically specific imaging reveals the distribution of O-linked sulfate esters. Because of the characteristic shifts in the edge position and intensity of different chemical forms of sulfur, multienergy chemically specific imaging can be performed to elucidate the distribution of specific chemical forms of sulfur (i.e, O-linked sulfate esters). In this example, two chemical forms of sulfur are revealed using a four-energy imaging approach. (A) H\&E stain. (B-E) Sulfur XFI maps at four specified energies: (B) $2475 \mathrm{eV}$, background; (C) $2478.2 \mathrm{eV}$, white line position of sulfonic acid; (D) $2479.8 \mathrm{eV}$, white line position of sulfate ester; (E) $2510 \mathrm{eV}$, total sulfur, displayed on a relative intensity scale. (F) Total phosphorus, measured simultaneously with sulfur. ( $\mathrm{G}$ and $\mathrm{H}$ ) Chemically specific images, displayed on a common intensity scale, of sulfonic acid and O-linked sulfate esters, respectively. (I) Overlay of total sulfur (red), total phosphorus (blue), and sulfate esters (green). Empty arrowheads indicate the locations of cartilage, and filled arrowheads indicate the locations of muscle tissue (intense pink eosin staining). The scale bar is $10 \mu \mathrm{m}$.

$2475 \mathrm{eV}$ the variations are substantially smaller, with intensity ranges (normalized units) of $0.087,0.049$, and 0.054 at 2475 , 2478.2 , and $2479.8 \mathrm{eV}$, respectively (Figure S5A). This range of intensity variance between 2475 and $2480 \mathrm{eV}$ is substantially smaller than the intensity in the region due to sulfate esters (Figure S5B). Likewise, micro-XAS spectra from wild-type and mutant cartilage typically differ by more than 0.6 across this region (Figure S5C). Therefore, although variation in relative compositions of thiols, thio-ethers, disulfides, and sulfoxides will produce small intensity variations across the energy range of $2475-2480 \mathrm{eV}$ that will not be fully subtracted by the background image at $2475 \mathrm{eV}$, the magnitude of these changes is 10 times less than the magnitude of the biochemical changes under investigation and thus does not alter the scientific outcome of the results. In addition, the relative composition of taurine and sulfate esters obtained with traditional linear regression of micro-XAS spectra corresponds well with our chemically specific imaging method (Figure S6).

FTIR Spectroscopic Imaging. Globar-FTIR-Focal Plane Array (FPA) spectroscopic images were collected at the Canadian Light Source (CLS) with a Hyperion 3000 microscope fitted with an upper objective of $36 \times$ magnification and a lower condenser of $15 \times$ magnification. This arrangement yielded a pixel size of $1.1 \mu \mathrm{m}$. The FTIRI data were collected with a spectral resolution of $4 \mathrm{~cm}^{-1}$ and the co-addition of 256 scans, and a background image was collected from blank substrate using 256 co-added scans. The background was collected immediately prior to each sample. The total protein content was calculated from the integrated area under the curve for the amide I band $\left(1700-1600 \mathrm{~cm}^{-1}\right)$, as previously reported for brain tissue. ${ }^{34,45,46,56,57}$ The ester carbonyl content was calculated from the integrated area under the curve from 1755 to $1715 \mathrm{~cm}^{-1}$.

All data processing and image generation were performed using Cytospec (Cytospec, version 1.2.04) and Opus (version 6.5, Bruker, Ettlingen, Germany).

Statistics. Three null hypotheses were tested. (1) Total sulfur and total phosphorus contents are not different between subcellular compartments (nucleus, cytosol, and cell membrane/extracellular matrix) $(n=3)$. (2) Cartilage maturation does not alter the total sulfur or sulfate ester content of cartilage (i.e., no significant difference exists between the anterior and medial regions of a cartilage element $)(n=5)$. (3)
Mutation of $f a m 20 b$ does not affect the total sulfur, sulfate ester, or elemental content of the cartilage relative to those of wild-type cartilage $(n=5)$. Values of $n$ in parentheses refer to the number of biological replicates within each group to test each null hypothesis. All samples to test null hypotheses 1 and 2 were paired samples and were tested with a paired Student's $t$ test. Hypothesis 3 was tested with a Student's $t$ test. All statistical analysis was two-tailed and used a 95\% confidence limit $(p<0.05)$. Statistical analysis was performed using SPSS 13.

\section{RESULTS AND DISCUSSION}

Development and Validation of Chemically Specific XFI. Sulfur K-edge X-ray absorption spectroscopy (XAS) spectra of inorganic sulfate, sulfonic acid, and $\mathrm{O}$ - and $\mathrm{N}$-linked sulfate esters are compared in Figure 2A. A number of other sulfur model compounds were also investigated (Table $S 1$ and Figure S1). While the exquisite sensitivity of the spectra of lower sulfur oxidation states has been reported previously, ${ }^{41,47}$ the higher-oxidation state species shown in Figure 2A also show remarkable sensitivity to chemical form. Inorganic sulfate $\mathrm{SO}_{4}{ }^{2-}$ has a characteristic single sharp absorbance at $2479.6 \mathrm{eV}$. This feature is attributable to a dipole-allowed $1 \mathrm{~s} \rightarrow 3 \mathrm{p}$ transition to the formally unoccupied $3 p$ manifold, with no obvious splitting because of $3 p$ degeneracy in the tetrahedral ligand field. ${ }^{48}$ For sulfate esters, breaking the tetrahedral symmetry causes a lifting of the $3 p$ degeneracy, with a corresponding broadened or split spectrum for $\mathrm{N}$ - or O-linked esters, respectively. ${ }^{48}$ Esters linked through $\mathrm{N}$ show a broadened peak at $2479.4 \mathrm{eV}$, while O-linked esters show a minor feature at $2478.5 \mathrm{eV}$ and an intense feature at $2479.8 \mathrm{eV}$. Hence, the pseudotetrahedral S(VI) compounds studied here can be distinguished spectroscopically.

Figure 2B-F shows both bulk and micro-XAS spectra of larval zebrafish tissue sections. Spectra were fitted to a linear combination of the full complement of model compounds to determine the relative sulfate ester contents; a representative example of this fitting is shown in Figure 2B. Histology was used to identify sections with high or low cartilage (Figure 2C), with bulk spectra averaging over sections rich in cartilage showing increased absorbance at 2478.5 and $2479.8 \mathrm{eV}$ (Figure 2D), consistent with O-linked sulfate esters. Similarly, microXAS (Figure 2F) of $5 \mu \mathrm{m}$ areas of cartilage, muscle, and eye lens (Figure 2E) showed significant differences in sulfur 
speciation for each tissue type. Thus, cartilage showed a predominance of O-linked sulfate esters; muscle showed high sulfonic acid (taurine) and protein thiol group contents, while eye lens showed only reduced forms from the methionine-rich lens crystallin proteins (Table S2). The major oxidized form and most spectroscopically prominent form of sulfur in cartilage matrix was O-linked sulfate ester; neither $\mathrm{N}$-linked sulfate esters nor inorganic sulfates were detected in any region. Chondroitin sulfate is the major proteoglycan molecule found in cartilage and contains approximately 50-100 chondroitin chains attached to a protein backbone, with each chain containing 20-30 sugar residues. ${ }^{49}$ In chondroitin sulfate, nearly all the sugar residues are thought to be sulfated and mostly bound as O-linked esters, ${ }^{49,50}$ and our data provide direct observation of this in cartilage.

Chemically specific XFI was used to image the relative distribution of sulfate esters in the larval zebrafish head (Figure $3)$. Because imaging at all excitation energies is timeconsuming, we employed a four-energy excitation approach, targeting only the oxidized forms that are the primary focus of this study. From the maps recorded at specific energies (Figure $3 \mathrm{~B}-\mathrm{E})$, background subtraction and matrix inversion are used to derive the maps of sulfonic acids (Figure 3G) and O-linked sulfate esters (Figure $3 \mathrm{H}$ ), along with total sulfur (Figure $3 \mathrm{E}$ ); total phosphorus (Figure $3 \mathrm{~F}$ ) is also recorded because the excitation energies are above the phosphorus K-edge. Notably, matrix inversion yields a sulfonic acid map with close to negligible intensities (Figure $3 \mathrm{G}$ ) even though the raw map at the sulfonic acid peak energy [2478.2 eV (Figure 3C)] shows substantial intensity; the latter can be attributed to the presence of sulfate esters. In particular, chemically specific XFI showed that although cartilage, retina, and muscle were rich in total sulfur (Figure 3E,I), sulfate esters were exclusively localized within cartilage (Figure $3 \mathrm{H}, \mathrm{I}$ ) and sulfonic acids were negligible throughout this section.

Cellular and Subcellular Localization of Sulfur in Chondrocytes. Figure 4 shows XFI of larval zebrafish cartilage at 1 and $0.1 \mu \mathrm{m}$ resolution (panels B and $\mathrm{C}$ and panels $\mathrm{E}$ and $\mathrm{F}$ of Figure 4, respectively). The bright field micrograph (Figure 4A) shows the location of chondrocytes and cell nuclei. As expected, the level of phosphorus is seen to be elevated in cell nuclei (Figure 4B,E). In contrast, each chondrocyte's periphery is enriched with sulfur (Figure 4C,F). The $0.1 \mu \mathrm{m}$ beam size used in the high-resolution images was insufficient to distinguish the cell membrane and extracellular matrix in this extremely small larval zebrafish cartilage. However, our imaging confirmed that the highest levels of sulfur are localized in the cell membrane or extracellular matrix (Figure 4D,F,G), in agreement with the expected distribution of sulfated PGs in cartilage. ${ }^{1,32,51}$ Our subcellular imaging revealed that while high sulfur levels exist within cartilage, some $40 \%$ of the total sulfur was localized within the cytoplasm and nucleus (Figure 4G). Thus, sulfated PGs are a major, but not the only, contributor to cartilage sulfur content, requiring careful consideration if total sulfur is used as an indirect marker of PG sulfation. Measurements of total sulfur at coarser spatial resolution, as has been done previously, ${ }^{19,21,52,53}$ will not differentiate sulfur located in the cell membrane and/or extracellular matrix from intracellular nuclear and cytoplasmic sulfur pools.

Relationship among Total Sulfur Levels, Sulfate Ester Levels, and Chondrocyte Maturation. We used XFI to investigate the hypothesis that cartilage maturation involves a decrease in total sulfur and sulfate ester content. As cartilage

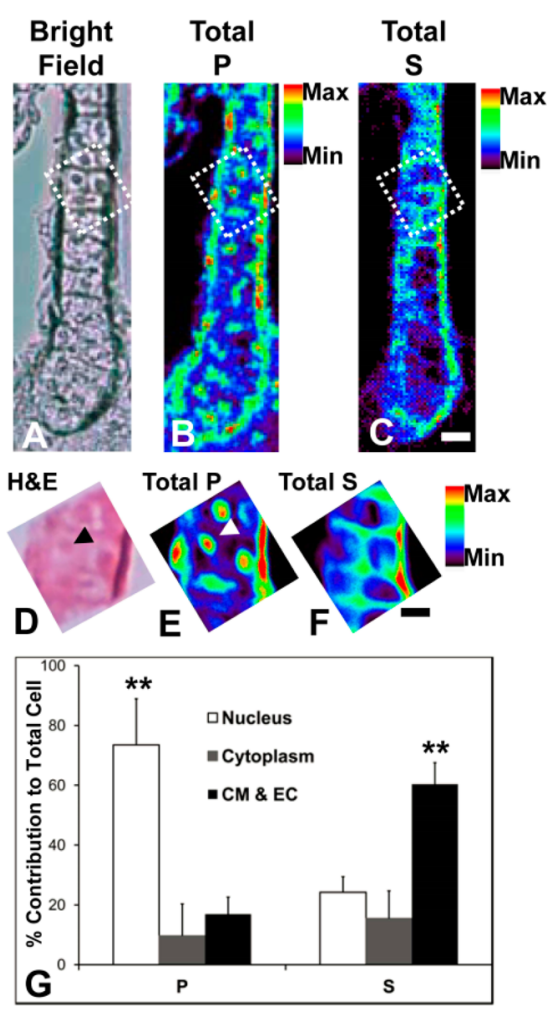

Figure 4. XFI of cartilage in larval zebrafish reveals subcellular compartmentalization. (A) Bright field imaging of the unstained cartilage prior to XFI analysis. (B and C) XFI elemental map of total phosphorus (P) and sulfur (S) distributions at $1 \mu \mathrm{m}$ steps within cartilage. (D) H\&E histology of the region analyzed at high resolution, which is outlined by a dashed line in panel A. (E and F) Highresolution (100 nm beam size and steps) XFI analysis of the P and S subcellular distribution in cartilage. (G) Quantification of $P$ and $S$ concentrations within subcellular compartments of cartilage (CM, cell membrane; EC, extracellular matrix). A Student's $t$ test at the $95 \%$ confidence limit was used to test for significant differences between groups. $* p<0.05$, and $* * p<0.01$. Scale bars are $10 \mu \mathrm{m}$ in panel $\mathrm{C}$ and $5 \mu \mathrm{m}$ in panel $\mathrm{F}$.

matures during endochondral ossification, mineralized bone begins to form in the adjacent tissue, the perichondrium. ${ }^{29}$ Therefore, to help define the region of mature cartilage in the XFI images, we used the high-magnitude phosphorus signal from adjacent perichondral bone. Because a high phosphorus level was observed in the midregion of the developing zebrafish ceratohyal (Figure 5B,E), consistent with known ossification patterns, ${ }^{32,54}$ we compared XFI of the midregion with XFI of the anterior and posterior regions. Both elemental XFI at the cellular level $[1 \mu \mathrm{m}$ resolution (Figure $5 \mathrm{~A}-\mathrm{C}$ ) $]$ and chemically specific XFI at the regional level $[5 \mu \mathrm{m}$ resolution (Figure $5 \mathrm{D}-$ $\mathrm{H})$ ] revealed that both total sulfur and sulfate ester levels were decreased in mature cartilage. Because overlap with muscle often occurred in the posterior region, quantitative analysis was performed only on medial versus anterior regions of the cartilage element. This demonstrated a significant decrease in the levels of both total sulfur and sulfate esters in the medial region, consistent with visual inspection of the images (Figure 5I), although this is subtle for the poorer spatial resolution XFI (Figure 5C compared with Figure 5I). The Supporting Information provides a discussion accounting for the differences between data sets obtained for the experiments performed at the different synchrotron facilities. 


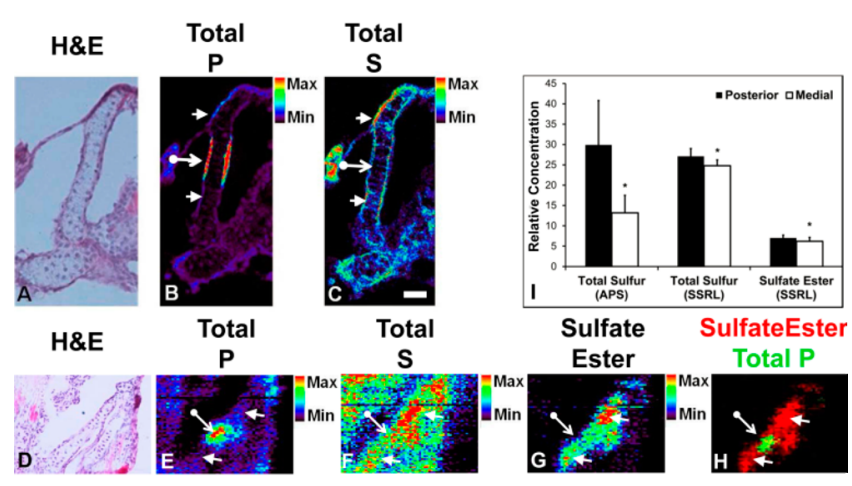

Figure 5. Phosphorus, sulfur, and sulfate ester distributions show significant variations during cartilage development. As development progresses, bone begins to form along the edge of cartilage, beginning in the middle segment. (A) H\&E histology of the cartilage imaged with XFI at the APS in panels B and C. (B) Total phosphorus (P) distribution highlighting a high $\mathrm{P}$ level in the middle of the cartilage element (long arrow) and a low $\mathrm{P}$ level at the posterior and anterior ends (short arrowheads). (C) Total sulfur (S) distribution showing low $S$ in the middle of the cartilage element (arrow) and a high $S$ level at the posterior and anterior ends (arrowheads). (D) H\&E histology of the cartilage element imaged with chemically specific XFI at SSRL (E-H). (E) Total P image from chemically specific XFI highlighting the high $\mathrm{P}$ level in the middle of the cartilage element (arrow) and the low P level at the posterior and anterior ends (arrowheads). (F) Total $S$ distribution showing the low $S$ level in the middle of the cartilage element (arrow) and the high $S$ level at the posterior and anterior ends (arrowheads). (G) Chemically specific XFI sulfate ester distribution showing the low sulfate ester content in the middle of the cartilage element (arrow) and the high sulfate ester content at the posterior and anterior ends (arrowheads). (H) Overlap of the P (green) and sulfate ester (red) distribution. (I) Quantitative analysis of the total S and sulfate ester content of posterior and medial cartilage regions. Total sulfur was measured at two different synchrotron facilities, the Advanced Photon Source (APS) and the Stanford Synchrotron Radiation Lightsource (SSRL), and the same scientific conclusion was reached in each case. The data are presented as the relative concentration (fluorescence yield). A Student's $t$ test at the $95 \%$ confidence limit was used to test for the significant difference between groups. ${ }^{*} p<0.05$. The scale bar is $10 \mu \mathrm{m}$.

Total Sulfur and Sulfate Ester Levels in PG-Defective Cartilage. Defective PG synthesis in fam 20 b mutant zebrafish, isolated from a mutagenesis screen, causes reduced levels of sulfated PGs in cartilage matrix. ${ }^{32,33}$ Indeed, the fam $20 b$ PG synthesis mutant shows a correlation between sulfated PG levels and chondrocyte differentiation, similar to that seen during normal development. XFI elemental analysis at the regional $(5 \mu \mathrm{m})$ level showed no significant changes between the wild-type and fam $20 b$ - mutant cartilage in terms of total sulfur and other elements ( $\mathrm{P}, \mathrm{Cl}, \mathrm{K}$, and $\mathrm{Ca}$ ) (Figure 6). Therefore, although the distributions of total sulfur and sulfate esters correlate with those of PGs in wild-type cartilage (Figure 5), cartilage from PG-defective mutant zebrafish does not have a decreased total sulfur content (Figure 6). This surprising result reinforces our earlier conclusion that total sulfur may not always be a reliable indicator of cartilage PG content. One possible cause for the lack of a decrease in total sulfur that would confound the scientific conclusions in this study is the possibility that tissue sections from mutant fish were cut slightly thicker than tissue sections from wild-type fish. The average total sulfur levels for data presented in Figure 6 are 10.55 \pm 2.3 and $10.88 \pm 1.91$ for wild-type and mutant fish, respectively. The $S / P$ ratios are $1.75 \pm 0.23$ and $1.90 \pm 0.16$ for wild-type

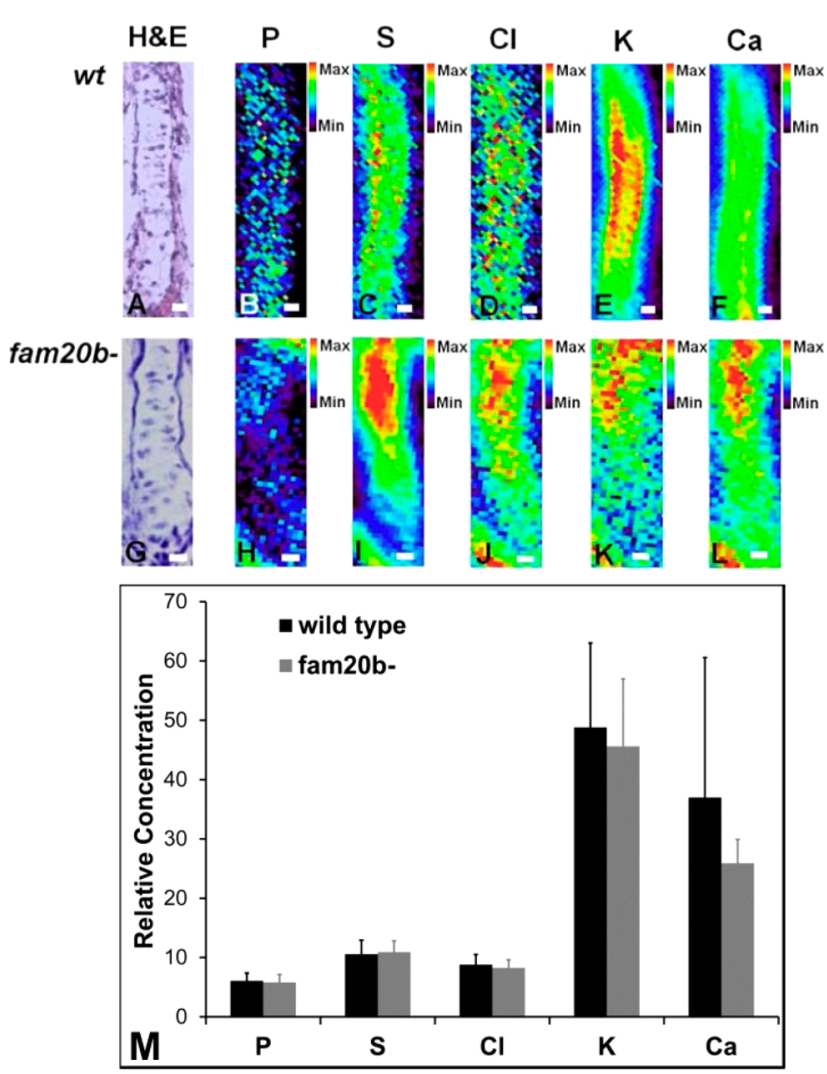

Figure 6. Mutation of PG synthesis has no significant effect on total elemental content. This figure compares the elemental levels and distribution in cartilage from wild-type (A-F) and fam $20 b$ - mutant $(\mathrm{G}-\mathrm{L})$ fish. After quantitation $(\mathrm{M})$, there were no differences in total sulfur [or any other element $(\mathrm{P}, \mathrm{Cl}, \mathrm{K}$, and $\mathrm{Ca})]$ from data collected at $3 \mu \mathrm{m}$ steps, in a $5 \mu \mathrm{m} \times 7 \mu \mathrm{m}$ beam. A Student's $t$ test at the $95 \%$ confidence limit was used to test for significant differences between groups. The scale bar is $5 \mu \mathrm{m}$. XFI elemental maps were collected at the CLS. The data are presented as relative concentration (fluorescence yield).

and mutant fish, respectively. The $\mathrm{S} / \mathrm{K}$ ratios are $0.22 \pm 0.052$ and $0.25 \pm 0.043$ for wild-type and mutant fish, respectively. Therefore, as the total sulfur, the $\mathrm{S} / \mathrm{P}$ ratio, and the $\mathrm{S} / \mathrm{K}$ ratio do not show any significant differences between mutant and wild-type fish, it is highly unlikely that variation in tissue thickness is masking a true decrease in total sulfur in mutant fish relative to wild-type fish.

Chemically specific XFI was used to reveal the sulfur speciation directly (Figure 7). This new technique confirmed our finding of no significant change in total sulfur but revealed a significant decrease in the O-linked sulfate ester content in fam 20b- cartilage, relative to that of the wild type (Figure $7 \mathrm{E}, \mathrm{J}, \mathrm{K})$. This difference was still significant in data normalized to total sulfur (Figure $7 \mathrm{~K}$ ), suggesting that the potential variation in tissue thickness or density was not a contributing factor. Indeed, the ratio of sulfate ester levels between wild-type and mutant fish (1.96) was within $10 \%$ of the sulfate ester/total sulfur ratio between wild-type and mutant fish (2.12), further supporting the finding that there is little variation in tissue thickness. To support these imaging results and to provide a broader analysis of all chemical forms of sulfur in wild-type and fam 20b- cartilage, micro-XAS spectra were collected and analyzed by linear combination curve fitting. The curve-fitting results show a subtle but significant decrease in sulfate ester 


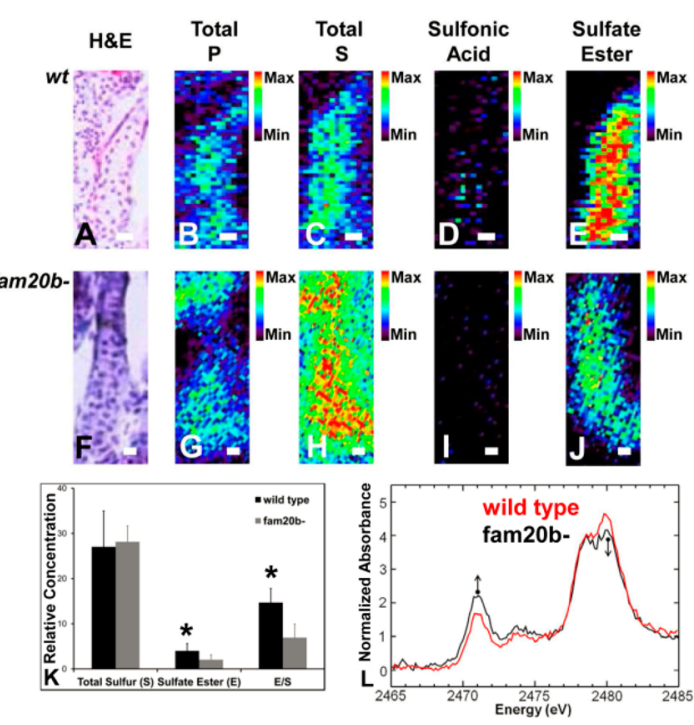

Figure 7. Chemically specific sulfur imaging shows a significant difference in sulfate ester, but not total sulfur, content in mutant (fam 20b-) cartilage compared with wild-type cartilage: (A-E) wild type and (F-J) fam 20b- mutant. (A and F) H\&E histology. (B and G) Total phosphorus. (C and H) Total sulfur. (D and I) Sulfonic acids. (E and $\mathrm{J}$ ) O-linked sulfate-esters. The scale bar is $5 \mu \mathrm{m}$. (K) Quantification of total sulfur and sulfate esters in cartilage. A Student's $t$ test at the $95 \%$ confidence limit was used to test for significant differences between groups. $* p<0.05$. The data are presented as relative concentration (fluorescence yield). (L) $\mu$-XAS of wild-type vs fam20b-cartilage, suggesting that while levels of sulfate esters are lower in the mutant (right arrow), levels of thiols and/or disulfides are higher (left arrow).

content in fam20b- cartilage relative to that of wild-type cartilage and more substantial changes in thiol and thio-ether contents (Figure 7L and Table S2). Future higher-spatial resolution XFI studies are expected to improve our understanding of the significance of these findings.

Total Protein Levels Are Increased in PG-Defective Cartilage. Fourier transform infrared (FTIR) spectroscopic imaging was used to determine the total protein content of wild-type and fam20b- PG-deficient cartilage (Figure 8). The results revealed that the fam $20 b$ - mutant fish have significantly increased total protein levels relative to that of wild-type fish. The levels of other absorbance bands (i.e., ester carbonyl band) were not significantly different between the wild-type and mutant fish, and the difference in total protein remained after normalization of the amide I band to the ester carbonyl band, an indication that variation in tissue thickness is not the cause of the differences in total protein.

\section{DISCUSSION}

Chemically specific sulfur K-edge XFI has been used previously to image sulfur speciation in situ in plants, ${ }^{24}$ rat brain, ${ }^{25}$ and bovine cornea; ${ }^{26}$ however, sulfate esters have remained largely unaddressed. In the initial plant study, sulfate esters were not substantially present in the tissues examined, while subsequent studies of brain ${ }^{25}$ and cornea ${ }^{26}$ incorrectly assumed that inorganic sulfate could be used to model sulfate esters, which we estimate could give quantitative errors exceeding $38 \%$ (Figure S2). Moreover, these studies ${ }^{25,26}$ did not use matrix inversion to solve for chemically specific images but instead used an analytically less rigorous difference method. Here we have further developed our methods ${ }^{24}$ to specifically image the distribution of sulfate esters in biological samples, providing the first direct in situ imaging of the distribution of sulfated proteoglycans in cartilage.

Our four-energy approach (e.g., Figure 3) neglects N-linked sulfate esters, which is reasonable because it is known that these are present only at low levels in cartilage; ${ }^{49,50}$ the majority (if not all) of the sulfate esters are present as sulfated PGs, ${ }^{1}$ with direct analysis of sulfate ester content reflecting the sulfated PG content. However, this will not be true for all tissue types. In brain, for example, a large component of sulfate esters exist as sulfatide lipids within white matter, as we have shown previously. ${ }^{36}$ Therefore, an accurate understanding of the chemical species likely to be present in each sample will be important when interpreting results. Future studies with higher spatial resolution may be able to identify discrete hot spots of $\mathrm{N}$-linked sulfate esters. With the development of new highintensity beamlines capable of operating at the sulfur K-edge with high-spatial and high-energy resolutions, we anticipate that imaging with a full complement of energies, both including lower oxidation states and resolving $\mathrm{O}$ - and $\mathrm{N}$-linked esters, will be routine.

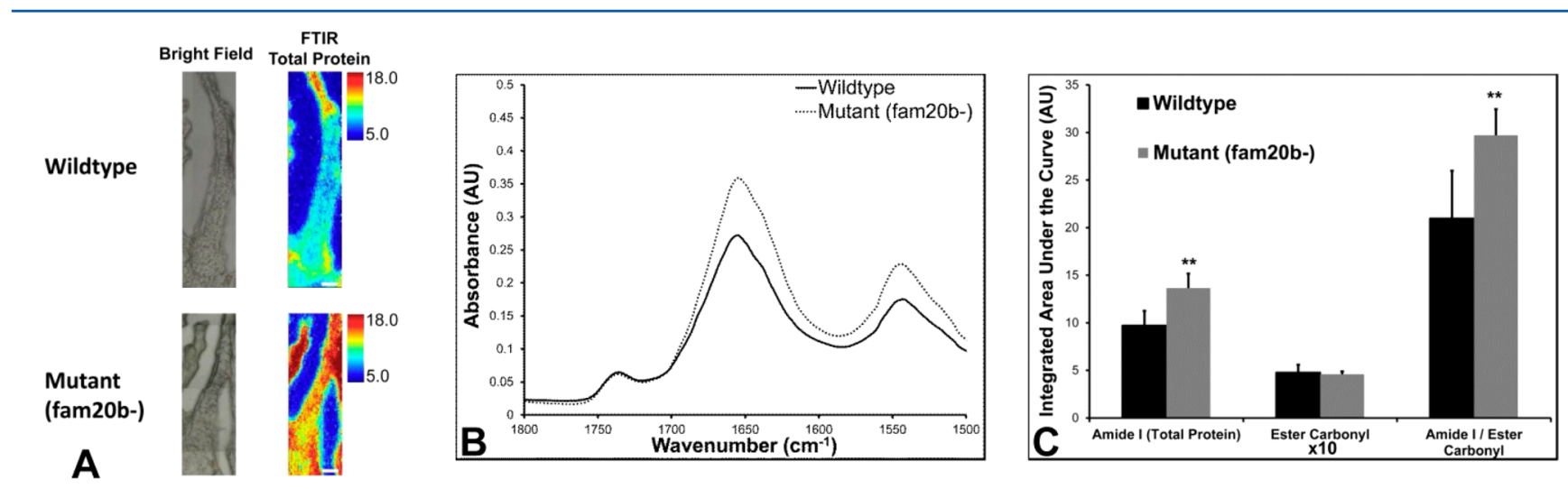

Figure 8. FTIR spectroscopic imaging reveals a significant increase in total protein levels in cartilage from PG-deficient fam20b- mutant fish relative to wild-type fish. (A) FTIR spectroscopic images of total protein in wild-type and fam20b-mutant fish were generated from the integrated area under the amide I band $\left(1600-1700 \mathrm{~cm}^{-1}\right)$. (B) Representative average FTIR spectra from the entire cartilage region of interest in one wild-type fish and one fam $20 b$ - mutant fish. (C) Average values calculated from the integrated area under the curve for the amide I band $\left(1600-1700 \mathrm{~cm}^{-1}\right)$, the ester carbonyl band $\left(1755-1715 \mathrm{~cm}^{-1}\right)$, and the amide I/ester carbonyl ratio. The integrated area under the curve values for the ester carbonyl band have been multiplied by a factor of 10 for the sake of clarity. 
Our findings that levels of total sulfur and sulfate esters decrease in the matrix of mature cartilage (Figure 5) are consistent with previous histological and biochemical work, ${ }^{30,31}$ and with nonimaging electron probe studies of tissue sections of growth plate cartilage of the chick ${ }^{20}$ and pig. ${ }^{53}$ Conversely, sulfur levels have been reported to increase again upon mineralization of cartilage. ${ }^{21,53}$ Because there is no mineralized cartilage at this stage of zebrafish development, we did not evaluate this. Our results do not support the finding that sulfur levels increase in hypertrophic cells of juvenile pig articular cartilage. ${ }^{52}$ The possibility that PG sulfation patterns may differ in growth plate and articular cartilage may be a focus in future studies, especially given the relevance of this distinction to diseases such as osteoarthritis.

An interesting conclusion from these data and previous work is that levels of PG sulfation somehow regulate cell differentiation. PG sulfation decreases during cartilage maturation, and cartilage maturation happens precociously when cartilage PG sulfation is reduced. ${ }^{32,55,56}$ Thus, the sulfation levels of cartilage PGs are inversely correlated with chondrocyte maturation. This observation calls into question the roles not only of PG synthesis genes but also of PG sulfation enzymes in regulating chondrocyte maturation. Two mechanisms can be proposed regarding the influence of sulfated PGs on gene expression: mechanical and biological. PG sulfation promotes extracellular matrix hydration, ${ }^{5}$ which provides cartilage matrix with mechanical properties that may regulate gene expression through mechanotransduction, likely via integrin signaling. ${ }^{57}$ PG sulfation also influences growth factor signaling, ${ }^{7-12}$ which is known to regulate gene expression. Further studies along these lines may inform our understanding of many human diseases, including osteoarthritis.

This study shows that total sulfur levels did not decrease in fam20b- PG-deficient fish, despite a significant decrease in sulfate ester levels. However, the micro-XAS spectra revealed that the content of lower-oxidation state sulfur species appeared to be increased in the mutant fish, which would account for the lack of a decrease in total sulfur content. A possible cause of the increase in lower-oxidation state sulfur could be an increased level of protein synthesis in the mutant fish, as part of a compensatory mechanism. To confirm this, FTIR spectroscopic imaging was performed to quantify the relative protein content in wild-type and mutant cartilage tissue. The results confirmed that the total protein levels are significantly increased in the mutant cartilage tissue, suggesting that total protein synthesis is different between wild-type and fam $20 b$ - mutant cartilage. Future work will now be conducted to determine the origins of the altered protein synthesis and if they act as a compensatory mechanism.

\section{CONCLUSIONS}

Here we have further developed chemically specific XFI and have applied it to cartilage to study the relationship between sulfated proteoglycans (PGs) and the differentiation program of cartilage cells. XFI shows that levels of both total sulfur and sulfate esters, the main biochemical form of sulfur in PGs, decrease as cartilage matures during endochondral ossification. Cartilage from a PG synthesis mutant also shows a subtle but significant decrease in sulfate esters relative to that of the wild type, providing genetic confirmation that the sulfate esters in developing cartilage observed by XFI are due to PG sulfation. In total, chemically specific XFI of developing normal and mutant cartilage reveals a functional correlation between PG sulfation and chondrocyte differentiation, potentially opening up a new avenue for exploring interactions between a cell and its extracellular matrix environment.

\section{ASSOCIATED CONTENT}

\section{(5) Supporting Information}

The Supporting Information is available free of charge on the ACS Publications website at DOI: 10.1021/acs.biochem.5b01136.

Supplementary Figures S1-S5 and Tables S1 and S2 (PDF)

\section{AUTHOR INFORMATION}

\section{Corresponding Authors}

*Telephone: 306-966-5722. Fax: 306-966-5683. E-mail: g. george@usask.ca.

*Telephone: 306-966-6534. E-mail: b.frank@usask.ca.

\section{Funding}

M.J.H. was supported by Saskatchewan Health Research Foundation (SHRF) and Canadian Institutes of Health Research (CIHR) postdoctoral fellowships, a CIHR-Training grant in Health Research using Synchrotron Techniques (CIHR-THRUST) fellowship, and the CIHR/Heart and Stroke Foundation of Canada (HSFC) Team in Synchrotron Medical Imaging of Stroke. I.J.P. and G.N.G. are supported by Canada Research Chair awards and by grants from the Natural Sciences and Engineering Research Council of Canada (NSERC), CIHR, SHRF, and the Saskatchewan Innovation and Science Fund. B.F.E. is supported by a SHRF Establishment Grant, an NSERC Discovery Grant, and a CIHR New Investigator Award. The CLS is supported by the Canada Foundation for Innovation, NSERC, the University of Saskatchewan, the Government of Saskatchewan, Western Economic Diversification Canada, the National Research Council of Canada, and CIHR. The APS is a U.S. Department of Energy (DOE) Office of Science (OS) User Facility operated for the DOE OS by Argonne National Laboratory under Contract DE-AC0206CH11357. SSRL, SLAC National Accelerator Laboratory, is supported by the U.S. DOE OS, Office of Basic Energy Sciences, under Contract DE-AC02-76SF00515. The SSRL Structural Molecular Biology Program is supported by the DOE Office of Biological and Environmental Research and by the National Institutes of Health (NIH), National Institute of General Medical Sciences (NIGMS) (including Grant P41GM103393).

\section{Notes}

The authors declare no competing financial interest.

\section{REFERENCES}

(1) Ham, A. W., Cormack, D. H. (1987) Ham's histology, 9th ed., Lippincott, Philadelphia.

(2) Pitsillides, A. A., and Beier, F. (2011) Cartilage biology in osteoarthritis-lessons from developmental biology. Nat. Rev. Rheumatol. 7, 654-663.

(3) Valdes, A. M., and Spector, T. D. (2010) The genetic epidemiology of osteoarthritis. Curr. Opin. Rheumatol. 22, 139-143.

(4) Chahine, N. O., Chen, F. H., Hung, C. T., and Ateshian, G. A. (2005) Direct measurement of osmotic pressure of glycosaminoglycan solutions by membrane osmometry at room temperature. Biophys. J. $89,1543-1550$.

(5) Otsuki, S., Hanson, S. R., Miyaki, S., Grogan, S. P., Kinoshita, M., Asahara, H., Wong, C. H., and Lotz, M. K. (2010) Extracellular 
sulfatases support cartilage homeostasis by regulating BMP and FGF signaling pathways. Proc. Natl. Acad. Sci. U. S. A. 107, 10202-10207. (6) Rosen, S. D., and Lemjabbar-Alaoui, H. (2010) Sulf-2: an extracellular modulator of cell signaling and a cancer target candidate. Expert Opin. Ther. Targets 14, 935-949.

(7) Cortes, M., Baria, A. T., and Schwartz, N. B. (2009) Sulfation of chondroitin sulfate proteoglycans is necessary for proper Indian hedgehog signaling in the developing growth plate. Development 136, 1697-1706.

(8) Gualeni, B., Facchini, M., De Leonardis, F., Tenni, R., Cetta, G., Viola, M., Passi, A., Superti-Furga, A., Forlino, A., and Rossi, A. (2010) Defective proteoglycan sulfation of the growth plate zones causes reduced chondrocyte proliferation via an altered Indian hedgehog signalling. Matrix Biol. 29, 453-460.

(9) Klüppel, M., Wight, T. N., Chan, C., Hinek, A., and Wrana, J. L. (2005) Maintenance of chondroitin sulfation balance by chondroitin4-sulfotransferase 1 is required for chondrocyte development and growth factor signaling during cartilage morphogenesis. Development 132, 3989-4003.

(10) Settembre, C., Arteaga-Solis, E., McKee, M. D., de Pablo, R., Al Awqati, Q., Ballabio, A., and Karsenty, G. (2008) Proteoglycan desulfation determines the efficiency of chondrocyte autophagy and the extent of FGF signaling during endochondral ossification. Genes Dev. 22, 2645-2650.

(11) Melrose, J., Isaacs, M. D., Smith, S. M., Hughes, C. E., Little, C. B., Caterson, B., and Hayes, A. J. (2012) Chondroitin sulphate and heparan sulphate sulphation motifs and their proteoglycans are involved in articular cartilage formation during human foetal knee joint development. Histochem. Cell Biol. 138, 461-475.

(12) Sohaskey, M. L., Yu, J., Diaz, M. A., Plaas, A. H., and Harland, R. M. (2008) JAWS coordinates chondrogenesis and synovial joint positioning. Development 135, 2215-2220.

(13) Barbosa, I., Garcia, S., Barbier-Chassefiere, V., Caruelle, J. P., Martelly, I., and Papy-Garcia, D. (2003) Improved and simple micro assay for sulfated glycosaminoglycans quantification in biological extracts and its use in skin and muscle tissue studies. Glycobiology 13, 647-653.

(14) Christner, J. E., Caterson, B., and Baker, J. R. (1980) Immunological determinants of proteoglycans. Antibodies against the unsaturated oligosaccharide products of chondroitinase ABCdigested cartilage proteoglycans. J. Biol. Chem. 255, 7102-7105.

(15) Pushie, M. J., Pickering, I. J., Korbas, M., Hackett, M. J., and George, G. N. (2014) Elemental and Chemically Specific X-ray Fluorescence Imaging of Biological Systems. Chem. Rev. 114, 84998541.

(16) Gomez, S., Rizzo, R., Pozzi-Mucelli, M., Bonucci, E., and Vittur, F. (1999) Zinc mapping in bone tissues by histochemistry and synchrotron radiation-induced $\mathrm{x}$-ray emission: correlation with the distribution of alkaline phosphatase. Bone 25, 33-38.

(17) Zoeger, N., Streli, C., Wobrauschek, P., Jokubonis, C., Pepponi, G., Roschger, P., Hofstaetter, J., Berzlanovich, A., Wegrzynek, D., Chinea-Cano, E., Markowicz, A., Simon, R., and Falkenberg, G. (2008) Determination of the elemental distribution in human joint bones by SR micro XRF. X-Ray Spectrom. 37, 3-11.

(18) Alaverdashvili, M., Hackett, M. J., Pickering, I. J., and Paterson, P. G. (2014) Laminar-specific distribution of zinc: Evidence for presence of layer IV in forelimb motor cortex in the rat. NeuroImage $103,502-510$.

(19) Cichocki, T., Gonsior, B., Hofert, M., Jarczyk, L., Raith, B., Rokita, E., Trzalkowski, A., and Sych, M. (1989) The analysis of mineral deposits and proteoglycans content in the cartilage of mouse trachea using PIXE in combination with proton microprobe. Acta Histochem. 85, 39-45.

(20) Hargest, T. E., Gay, C. V., Schraer, H., and Wasserman, A. J. (1985) Vertical distribution of elements in cells and matrix of epiphyseal growth plate cartilage determined by quantitative electron probe analysis. J. Histochem. Cytochem. 33, 275-286.

(21) Reinert, T., Reibetanz, U., Vogt, J., Butz, T., Werner, A., and Gründer, W. (2001) Spatially resolved elemental distributions in articular cartilage. Nucl. Instrum. Methods Phys. Res., Sect. B 181, 516521.

(22) Rizzo, R., Grandolfo, M., Godeas, C., Jones, K., and Vittur, F. (1995) Calcium, sulfur, and zinc distribution in normal and arthritic articular equine cartilage: A synchrotron radiation-induced X-ray emission (SRIXE) study. J. Exp. Zool. 273, 82-86.

(23) Vittur, F., Tuniz, C., Psoletti, S., Rizzo, R., and Jones, K. W. (1992) Elemental analysis of growth plate cartilage by synchrotronradiation-induced X-ray emission (SRIXE). Biochem. Biophys. Res. Commun. 188, 1010-1017.

(24) Pickering, I. J., Sneeden, E. Y., Prince, R. C., Block, E., Harris, H. H., Hirsch, G., and George, G. N. (2009) Localizing the Chemical Forms of Sulfur in Vivo Using X-ray Fluorescence Spectroscopic Imaging: Application to Onion (Allium cepa) Tissues. Biochemistry 48, 6846-6853.

(25) Szczerbowska-Boruchowska, M., Stegowski, Z., Lankosz, M., Szpak, M., and Adamek, D. (2012) A synchrotron radiation micro-Xray absorption near edge structure study of sulfur speciation in human brain tumors-a methodological approach. J. Anal. At. Spectrom. 27, 239-247.

(26) Veronesi, G., Koudouna, E., Cotte, M., Martin, F. L., and Quantock, A. J. (2013) X-ray absorption near-edge structure (XANES) spectroscopy identifies differential sulfur speciation in corneal tissue. Anal. Bioanal. Chem. 405, 6613-6620.

(27) Ali, S., Champagne, D. L., Spaink, H. P., and Richardson, M. K. (2011) Zebrafish embryos and larvae: a new generation of disease models and drug screens. Birth Defects Res., Part C 93, 115-133.

(28) Zon, L. I. (1999) Zebrafish: a new model for human disease. Genome Res. 9, 99-100.

(29) Eames, B. F., de la Fuente, L., and Helms, J. A. (2003) Molecular Ontogeny of the Skeleton. Birth Defects Res., Part C 69, 93101.

(30) Deutsch, A. J., Midura, R. J., and Plaas, A. H. (1995) Structure of chondroitin sulfate on aggrecan isolated from bovine tibial and costochondral growth plates. J. Orthop. Res. 13, 230-239.

(31) Farquharson, C., Whitehead, C. C., and Loveridge, N. (1994) Alterations in glycosaminoglycan concentration and sulfation during chondrocyte maturation. Calcif. Tissue Int. 54, 296-303.

(32) Eames, B. F., Yan, Y. L., Swartz, M. E., Levic, D. S., Knapik, E. W., Postlethwait, J. H., and Kimmel, C. B. (2011) Mutations in fam20b and xylt1 reveal that cartilage matrix controls timing of endochondral ossification by inhibiting chondrocyte maturation. PLoS Genet. 7, e1002246.

(33) Miller, M. R., Atwood, T. S., Eames, B. F., Eberhart, J. K., Yan, Y. L., Postlethwait, J. H., and Johnson, E. A. (2007) RAD marker microarrays enable rapid mapping of zebrafish mutations. Genome Biol. 8, R105.

(34) Hackett, M. J., Borondics, F., Brown, D., Hirschmugl, C., Smith, S. E., Paterson, P. G., Nichol, H., Pickering, I. J., and George, G. N. (2013) A Sub-Cellular Biochemical Investigation of Purkinje Neurons Using Synchrotron Radiation Fourier Transform Infrared Spectroscopic Imaging with a Focal Plane Array Detector. ACS Chem. Neurosci. 4, 1071-1080.

(35) Hackett, M. J., McQuillan, J. A., El-Assaad, F., Aitken, J. B., Levina, A., Cohen, D. D., Siegele, R., Carter, E. A., Grau, G. E., Hunt, N. H., and Lay, P. A. (2011) Chemical alterations to murine brain tissue induced by formalin fixation: implications for biospectroscopic imaging and mapping studies of disease pathogenesis. Analyst 136, 2941-2952.

(36) Hackett, M. J., Smith, S. E., Paterson, P. G., Nichol, H., Pickering, I. J., and George, G. N. (2012) X-ray Absorption Spectroscopy at the Sulfur K-Edge: A New Tool to Investigate the Biochemical Mechanisms of Neurodegeneration. ACS Chem. Neurosci. 3, 178-185.

(37) Gnida, M., Yu Sneeden, E., Whitin, J. C., Prince, R. C., Pickering, I. J., Korbas, M. g., and George, G. N. (2007) Sulfur X-ray Absorption Spectroscopy of Living Mammalian Cells: An Enabling Tool for Sulfur Metabolomics. In Situ Observation of Uptake of Taurine into MDCK Cells. Biochemistry 46, 14735-14741. 
(38) Pickering, I. J., George, G. N., Yu, E. Y., Brune, D. C., Tuschak, C., Overmann, J., Beatty, J. T., and Prince, R. C. (2001) Analysis of sulfur biochemistry of sulfur bacteria using X-ray absorption spectroscopy. Biochemistry 40, 8138-8145.

(39) Sekiyama, H., Kosugi, N., Kuroda, H., and Ohta, T. (1986) Sulfur K-edge absorption spectra of $\mathrm{Na} 2 \mathrm{SO} 4, \mathrm{Na} 2 \mathrm{SO} 3, \mathrm{Na} 2 \mathrm{~S} 2 \mathrm{O} 3$, and Na2S2Ox $(\mathrm{x}=5-8)$. Bull. Chem. Soc. Jpn. 59, 575-579.

(40) George, M. J. (2000) XAS-Collect: A computor program for Xray absorption spectroscopic data acquisition. J. Synchrotron Radiat. 7 , 283-286.

(41) Pickering, I. J., Prince, R. C., Divers, T., and George, G. N. (1998) Sulfur K-edge X-ray absorption spectroscopy for determining the chemical speciation of sulfur in biological systems. FEBS Lett. 441, $11-14$.

(42) George, G. N. http://ssrl.slac..stanford.edu/exafspak.html.

(43) Webb, S. M. (2011) The MicroAnalysis Toolkit: X-ray Fluorescence Image Processing Software. In The 10th International Conference on X-ray Microscopy, pp 196-199, AIP Publishing.

(44) Vogt, S. (2003) MAPS: A set of software tools for analysis and visualization of 3D X-ray fluorescence data sets. J. Phys. IV 104, 635638 (EDP sciences).

(45) Hackett, M. J., Caine, S., Liu, X., May, T. E., and Borondics, F. (2015) Development of single-beam wide-field infrared imaging to study sub-cellular neuron biochemistry. Vib. Spectrosc. 77, 51-59.

(46) Hackett, M. J., Smith, S. E., Caine, S., Nichol, H., George, G. N., Pickering, I. J., and Paterson, P. G. (2015) Novel bio-spectroscopic imaging reveals disturbed protein homeostasis and thiol redox with protein aggregation prior to hippocampal CA1 pyramidal neuron death induced by global brain ischemia in the rat. Free Radical Biol. Med. 89, 806-818.

(47) Rompel, A., Cinco, R. M., Latimer, M. J., McDermott, A. E., Guiles, R. D., Quintanilha, A., Krauss, R. M., Sauer, K., Yachandra, V. K., and Klein, M. P. (1998) Sulfur K-edge X-ray absorption spectroscopy: a spectroscopic tool to examine the redox state of $S$ containing metabolites in vivo. Proc. Natl. Acad. Sci. U. S. A. 95, 61226127.

(48) Frank, P., Hedman, B., and Hodgson, K. O. (1999) Sulfur Allocation and Vanadium-Sulfate Interactions in Whole Blood Cells from the Tunicate Ascidia ceratodes, Investigated Using X-ray Absorption Spectroscopy. Inorg. Chem. 38, 260-270.

(49) Muir, H. (1978) Proteoglycans of cartilage. J. Clin. Pathol. s3-12, 67.

(50) Mikami, T., and Kitagawa, H. (2013) Biosynthesis and function of chondroitin sulfate. Biochim. Biophys. Acta, Gen. Subj. 1830, 47194733.

(51) Schaefer, L., and Schaefer, R. M. (2010) Proteoglycans: from structural compounds to signaling molecules. Cell Tissue Res. 339, 237-246.

(52) Reinert, T., Reibetanz, U., Schwertner, M., Vogt, J., Butz, T., and Sakellariou, A. (2002) The architecture of cartilage: Elemental maps and scanning transmission ion microscopy/tomography. Nucl. Instrum. Methods Phys. Res., Sect. B 188, 1-8.

(53) Althoff, J., Quint, P., Krefting, E. R., and Hohling, H. J. (1982) Morphological studies on the epiphyseal growth plate combined with biochemical and X-ray microprobe analyses. Histochemistry 74, 541552.

(54) Eames, B. F., DeLaurier, A., Ullmann, B., Huycke, T. R., Nichols, J. T., Dowd, J., McFadden, M., Sasaki, M. M., and Kimmel, C. B. (2013) FishFace: interactive atlas of zebrafish craniofacial development at cellular resolution. BMC Dev. Biol. 13, 23.

(55) Domowicz, M. S., Cortes, M., Henry, J. G., and Schwartz, N. B. (2009) Aggrecan modulation of growth plate morphogenesis. Dev. Biol. 329, 242-257.

(56) Mis, E. K., Liem, K. F., Jr, Kong, Y., Schwartz, N. B., Domowicz, M., and Weatherbee, S. D. (2014) Forward genetics defines Xylt1 as a key, conserved regulator of early chondrocyte maturation and skeletal length. Dev. Biol. 385, 67-82.
(57) Kim, S.-H., Turnbull, J., and Guimond, S. (2011) Extracellular matrix and cell signalling: the dynamic cooperation of integrin, proteoglycan and growth factor receptor. J. Endocrinol. 209, 139-151. 